\title{
High-Throughput Sequencing Technologies and new Approaches in Food Microbiology
}

\author{
Tiziana Maria Sirangelo \\ Department of Life Sciences, University of Modena and Reggio Emilia, Modena, Italy.
}

*Corresponding Author: Tiziana Maria Sirangelo, Department of Life Sciences, University of Modena and Reggio Emilia, Modena, Italy.

\begin{abstract}
Recent investigation approaches, based on High-Throughput Sequencing (HTS) technologies, allow to develop in-depth analysis of food microbiota/microbiome. HTS, which identify the modern Next Generation Sequencing (NGS) techniques, contribute to food investigation advances by allowing to produce a larger volume of data at a price that is decreasing. This contribute offers an overview about the latest and most innovative techniques for analyzing food microbiota. Furthermore, the impact on food microbiology of omics approaches based on HTS is discussed. Relevant metagenomics, metatranscriptomics, metaproteomics studies concerning different food application fields are treated. The current scenario and pregnant omics and multi-omics works are highlighted, showing the usefulness to adopt an integrated multi-omics approach in different food microbiology areas, especially in food quality and safety. In this context, nowadays, food is more and more considered not only as a source of energy but also as an affordable way to prevent diseases. Finally, some technical limitations of multi-omics methods are outlined. If in the past an integrative omics approach was not feasible because of the lack of expertise and technical limitations, the current technological advances offer high performances in terms of standardization to investigate a wide data set coming different platforms.
\end{abstract}

Keywords: food microbiology, high-throughput sequencing, next generation sequencing, omics technologies, multi-omics approach

\section{INTRODUCTION}

In relatively recent years, relevant changes in the approaches used for food microbiological analysis occurred. The PCR (Polymerase Chain Reaction) method [1] has led to new strategies to study food microbiota and it has become possible to investigate microorganisms without that any cultivation approach is used.

Culture dependent methods have been characterized by laborious and time-consuming procedures and allows generally to recovery less than $20-30 \%$ of total bacteria richness [2]. The introduction of culture independent methods has allowed to understand and to overcome the limitation of microbial cultivation [3]. It was applied in food microbiology as tools to analyze foodstuff microbiota, to study fermentation and food spoilage processes and also to investigate the ecology of food-borne pathogens $[4,5]$.

In PCR-based methods, DNA, RNA and proteins are the preferred targets. After amplification of the nucleic acids extracted directly from the food matrix, the PCR product is subjected to specific analyses in order to highlight differences in the amplified DNA sequences [6]. In this approach, the selection of the DNA region to be amplified is very important. The most frequently regions used in bacteria are related to the 16S rRNA coding gene, while the 26S rRNA (or Internal Transcribed Spacer, ITS) is generally the target in yeasts. For these two genes large sequence databases have been put in place.

In the last years the introduction of new methodologies in studying food microbiota occurred. These methodology, called next generation sequencing (NGS), has a great advantage respect the previous analysis methods, in which only a part of the considered food sample microbiota can be identified. In fact, NGS allows to obtain in relatively short time a massive quantity of sequences from a single run cycle [7]. 


\section{OMICS SCIENCES}

The dissemination of omics sciences, that encompass several approaches, as metagenomics, metatranscriptomics, as well as metaproteomics and metabolomics, has been made possible mainly thanks to the development of NGS technologies. These approaches can generate huge amounts of data with different levels of biological complexity, (DNA, mRNA, protein, metabolites, etc.).

Metagenomics allows the analysis of whole-genome sequences of DNA extracted from an organism, in which bioinformatic tools are used to individuate differences between the sequences [8]. It is an extremely powerful method that can be adopted to describe the genetic potential of several kinds of microbiome, and includes two fundamental approaches: the amplicon sequencing method, that uses the high-throughput 16S rRNA gene regions, and the shoutgun method which concerns the coverage of the genome outside of the small 16S rRNA gene region and this means that specific, strain level discrimination is achievable [9]. The last approach covering the entire genome of an organism and skips the amplification step.

Figure 1 shows the differences between these two methods.
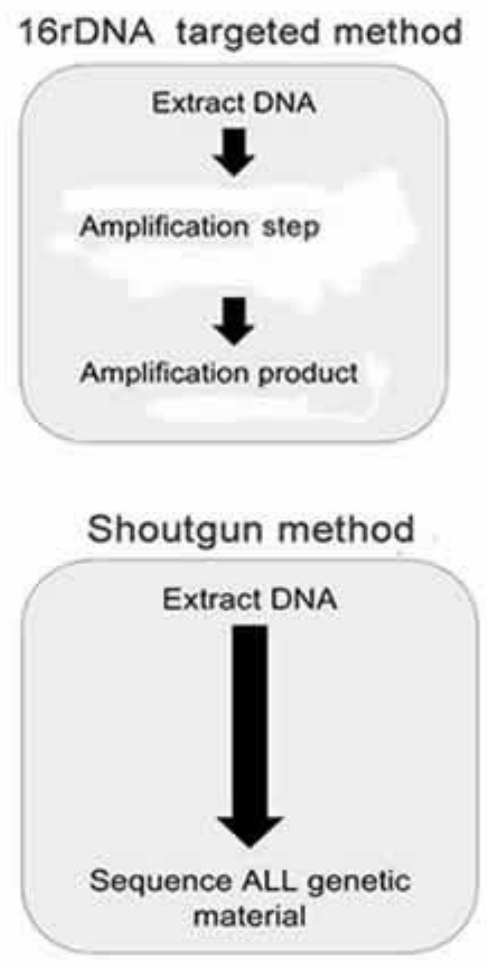

Figure 1. targeted 16rDNA and shoutgun methods.

Metatranscriptomics is based on the retrieval and sequencing of mRNAs from a microbial ecosystem and its focus is on the genes activity. It requires high-depth sequencing to detect differentially expressed transcripts present in low abundances, consequently, metatranscriptomics is one of the most expensive HTS approaches [10].

Metaproteomics allows the analysis of protein to understand the functions of the microbial community. It may be defined as "the large-scale characterization of the entire protein complement of environmental microbiota at a given point in time" [11].

Metabolomics allows to define the metabolic profile of different microbiome, including food microbiome [12]. It refers to the systematic identification and quantification of the small molecule metabolic products (the metabolome) of a biological system (as cell, tissue, organ) at a specific point in time. Mass spectrometry (MS) and Nuclear Magnetic Resonance (NMR) spectroscopy are the techniques most often used in metabolomics. A good advantage of both techniques is the highthroughput capability of spectroscopic and structural information allowing to characterize a wide range of metabolites simultaneously, with high analytical precision. Compared to NMR, MS is more sensitive and provide information for metabolites present at low concentrations. 
All these omics approaches have been applied in food microbiology.

\section{APPLICATIONS OF NEXT GENERATION SEQUENCING IN FOOD}

Over the past two decades, omics technologies have dramatically changed biological research, and the focus has been on advances in DNA sequencing methods [7]. Food microbiology benefited from advances occurred in molecular biology and new techniques to detect and monitor microorganisms have been developed.

Figure 2. shows some food application fields based on modern omics analytical techniques.

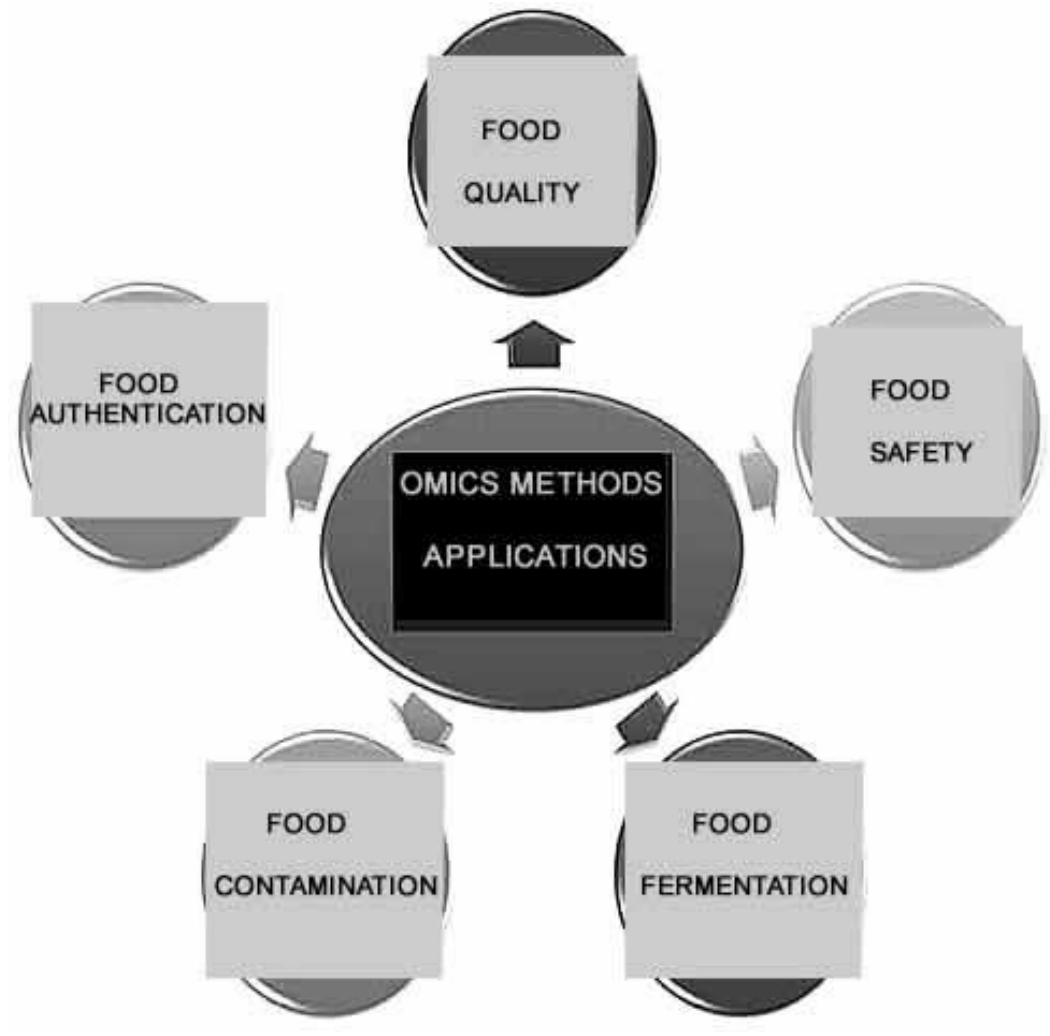

Figure 2. Application of omics studies in food microbiology fields

Innovative applications in food quality and safety are discussed in the following sections.

\subsection{Food Quality}

Consumers have nowadays a considerable attention to quality of foodstuffs and food ingredients. Products must have exhaustive labelling reporting details describing the original raw materials and the absence of harmful chemical and contaminants [13].

Pathogens can be present in the initial microbiota or colonize the processed product by contamination during the food manufacturing activities. Salmonella spp. is one of the major pathogens responsible for foodborne disease outbreaks and S.enterica is the most frequently isolated species [14]. Other frequently foodborne pathogens belong to the genera Campylobacter, Yersinia, Shigella, Vibrio, Clostridium, Bacillus, Listeria, and Staphylococcus has been reported [15].

Relevant approaches was devoted to screen undesired microorganisms in foodstuffs and to prevent the spread of foodborne disease outbreaks [14]. Most of these pathogens are not easily individuated by using traditional approaches, and metagenomic methods have been adopted, mainly using 16s rDNA or $26 \mathrm{~s}$ rDNA gene sequencing $[16,17]$. These approaches have a growing importance also in the authentication product activity [18]. In fact, the food market globalization has led a comprehensive food supply chain, where raw materials are often processed in different countries. At the same time, the industrialization of manufacturing processes are more complex and widely unknown to the consumers [19].

The availability of advanced technologies and of commercial kits for DNA extraction allows to obtain genetic material from biological material. However, the DNA certification is not necessarily 
synonyms of food quality [13]. In fact, environmental factors could affect the food organoleptic properties [20] and, for this reason, the DNA-based approach should be combined with chemical food studies. Therefore, the analysis of food metabolome is essential in the evaluation of food quality, in fact metabolites are the final downstream products of the genome and of its interactions with the environment [21]. Recently, some investigations have analyzed as the level of ripening of some foods, as peach, depends on the abundance of several metabolites, including amino acids and sugars [22]. Examples of metabolic approaches on different foodstuffs include grape and wine [23].

The advantages of DNA analysis and metabolomics integration are relevant and would constitute a good approach in food quality. One example is that of wine, which can be studied with both DNA analysis of the grape and the metabolic identification of its characteristics, such as fermentation behaviours and antioxidant properties.

The analysis of metabolome has been used in identifying specific chemical items related to the geographic production area [24]. The origin of wine could also be supported by the DNA-based analysis of must/wine microbiome [25].

An important aspect of food metabolome is the flavour and aroma determination. The volatile metabolomics pattern of beer raw materials has been defined in a recent paper [26]. Similar results were obtained with aromatic spices [27]. Furthermore, the integration of metabolomics technique with other technologies is important to allow the link among microbial metabolites and sensorial attributes [28].

DNA based approaches combined with the use of HTS technologies could provide great advantages in this food quality because it allows to obtain a good overview of food pathogens [13].

Recently is known the need to address the food analysis by the exploitation of integrative omics approaches. A recent study focusing on cheese ripening is an example in which data coming from metagenomic, metatranscriptomic and biochemical analyses have been combined to obtain an overall view of what happens during this process [29]. Another research showed that coupling metatranscriptomic and metabolome data is effective in discovering the functional diversity of cheese microbiota affected by different ripening conditions [30] .

Furthermore, HTS techniques have been applied to several samples of ricotta to evaluate the microbial community composition in depth using both RNA and DNA as templates for NGS library construction [31]. An integrated analysis based on multi-omics approach gave us the opportunity to better understand how plants modulate their response to different environments [32]. Omics techniques has been applied to detect also food authenticity and could be implemented to ensure food integrity [18]. For instance, a multi-omics method to analyze metabolites, endogenous oligosaccharides, proteins and peptides, has been used to find Biomarkers in Philippine Civet Coffee [33].

Lastly, the correlation of data coming from different omics techniques, in a multi-omics approach, would contribute to the overall quality of food products.

\subsection{Food Safety}

The importance of foodborne pathogens and the development of antimicrobial resistance have been recognized as fundamental issues to food safety and health. In fact, new safety risks have to be taken into consideration because of the continuous adaptation of relevant pathogens, changes in food production methodologies and in the environment. Other factors which have contribute to the increasing of foodborne diseases are the food supply globalization, the growing international food trade and travel, changes in agricultural practices. Consequently, an increasing number of food is considered as potential vehicle for illnesses.

At the same time, the interest to investigate fungal toxins, toxic chemicals and resulting changes during food production processes is improving. There are a shift from traditional food problems which were mostly of animal origin such as meat, eggs, to problems regarding fresh food of plant origin, and traditional fermented food products [34]. This has led the development of new methods for identifying hazards during food production, storage and delivery.

Several omics methods such as DNA based techniques, spectroscopy, sensory methods and bioimaging have been used in food safety [35]. These techniques play a relevant role in the 
identification of antibiotics in food products and in the evaluation of adulteration of raw materials in production. It has been demonstrated that effective methods of food analysis and detection of adulterants can be further optimized through high-throughput methods [36-38]. Compared to traditional methods, these technologies are sensitive and quick to generate information [39].

Particularly, the whole genome sequencing (shoutgun) as a tool for subtyping of pathogen isolates represents considerable potential for improving the rapid detection of disease outbreaks [40]. A relevant application based this metagenomic approach concerns the detection of foodborne pathogens $[41,42]$. Other metagenomics applications in food safety include the identification of novel and nonculturable agents that cause disease [43].

In food safety area, integrated omics approaches have been also used. In detail, integrated metagenomics and metabolomics methods have been adopted to enhance the safety of fermented foods to identify microorganisms producing harmful metabolites. For this aim, 16S rRNA and metabolomics were used to detect biogenic amines in Chinese rice wine [44]. Likewise the safety of other fermented foods has been improved [45]. Furthermore, to study the fermentation process of kombucha tea, ITS and metabolomics were combined to demonstrate that fungal population evolutions was related to the improvement antioxidants levels [46]. Recent investigations have assessed transcriptomes and/or proteomes of bacteria under particular conditions, for instance, simulating the behavior of $E$. coli during beef carcass chilling [47]. Several studies have been demonstrated that an integrated approach based on transcriptomics and proteomics to be utilized for a rational development of new control strategies for foodborne pathogens [40].

As in food quality area, correlation of data coming from different omics techniques, would contribute to improve safety of food products.

\section{CONCLUSIONS AND DISCUSSION}

As it is evident from what was discussed in the previous section, the future of food microbiology analysis will be necessarily based on the exploitation of omics platforms and specially of multi-omics platforms. This it is mostly essential to obtain an exhaustive characterization of food quality and safety or to assess the effect of food on human cells and tissues. The availability of such platforms responds to a general trend in food science about the linking between food and health. Nowadays, food is more and more considered not only as a source of energy but also as an affordable way to prevent future diseases.

In this scenario, a new discipline known as "foodomics" has been defined to study the food and nutrition domains through the application of advanced omics technologies to improve consumer's well-being [48].

For example, a foodomics analysis was used to evaluate the effect of dietary polyphenols against human colon cancer [49], in which has been tested the chemo-preventive effect of polyphenols from rosemary on the total gene, protein, and metabolite expression in colon cancer cells. The genes, proteins, and metabolites involved in this process were identified thanks to the multi-parameter omics analysis.

If in the past an integrative omics approach was not feasible because of the lack of expertise and technical limitations [50], the current technological advances offer high performances in terms of standardization to investigate a wide set of food items. In fact, we may consider that multi-omic approaches provide biological analysis coming from datasets that are usually large. An initial power calculation to ensure sufficient sample size is thus necessary in large-scale studies. In addition a detailed data analysis is necessary in order to define and develop a computational pipeline able to integrate data generated from different platforms.

We believe that the spread of omics platforms will be able to simultaneously process different food matrices with a multi-approach strategy, which will be made unified and standardized under the control of bioinformatics tools.

\section{REFERENCES}

[1] K. Mullis, F. Faloona, S. Scharf, R. Saiki, G. Horn, H. Erlich, Specific enzymatic amplification of DNA in vitro: the polymerase chain reaction, Cold Spring Harbor Symposium on Quantitative Biology, vol. 51, pp. 263-373, (1986). 
[2] R. Temmerman, G. Huys, J. Swings, Identification of lactic acid bacteria: culture-dependent and cultureindependent methods, Trends in Food Science \& Technology, vol. 15, pp. 348-359, (2004).

[3] L. Cocolin, D. Ercolini, Molecular Techniques in the Microbial Ecology of Fermented Foods, Springer, (2008).

[4] L. Cocolin, K. Rantsiou, L. Iacumin, C. Cantoni, G. Comi, Direct identification in food samples of Listeria spp. and Listeria monocytogenes by molecular methods, Applied and Environmental Microbiology, vol. 68, pp. 6273-6282, (2002).

[5] L. Cocolin, G. Comi, Use of a culture-independent molecular method to study the ecology of Yersinia spp. in food, International Journal of Food Microbiology, vol. 105, pp. 71-82, (2005).

[6] L. Cocolin, V. Alessandria, P. Dolci, R. Gorra, K. Rantsiou, Culture independent methods to assess the diversity and dynamics of microbiota during food fermentation, International Journal of Food Microbiology, vol. 167, pp. 29-43, (2013).

[7] D.C. Koboldt, K.M. Steinberg, D.E. Larson, R.K. Wilson, E.R. Mardis, The next-generation sequencing revolution and its impact on genomics, Cell, vol. 155, pp.27-38, (2013).

[8] J. Herrero, M. Muffato, K. Beal, S. Fitzgerald, L. Gordon, et al. Ensembl comparative genomics resources, Database (Oxford), (2016).

[9] E.A. Franzosa, T. Hsu, A. Sirota-Madi, A. Shafquat, G. Abu-Ali, et al., Sequencing and beyond: integrating molecular 'omics' for microbial community profiling, Nature Reviews Microbiology, vol. 13, pp. 360-72, (2015).

[10] R. Lei, K. Ye, Z. Gu, X. Sun, Diminishing returns in next-generation sequencing (NGS) transcriptome data, Gene, vol. 557, pp. 82-87, (2015).

[11] P. Wilmes, A. Heintz-Buschart, P.L. Bond, A decade of metaproteomics: Where we stand and what the future holds, Proteomics, (2015).

[12] S. O'Flaherty, T.R. Klaenhammer, The impact of omic technologies on the study of food microbes, Annu Rev Food Sci Technol., vol. 2, pp. 353-71, (2011).

[13] E. Ferri, A. Galimberti, M. Casiraghi, C. Airoldi, C.Ciaramelli, A. Palmioli, V.Mezzasalma, I. Bruni, and M. Labra, Towards a Universal Approach Based on Omics Technologies for the Quality Control of Food, BioMed Research International, vol.2015, Article ID 365794, pp. 14, (2015).

[14] B. R. Jackson, P. M. Griffin, D. Cole, K. A. Walsh, and S. J. Chai, Outbreak-associated Salmonella enterica serotypes and food commodities, United States, 1998-2008, Emerging Infectious Diseases, vol. 19, no. 8, pp. 1239-1244, (2013).

[15] C.J. Doyle, D. Gleeson, P.W.O'Toole, P.D. Cotter, High-throughput metataxonomic characterization of the raw milk microbiota identifies changes reflecting lactation stage and storage conditions, International Journal of Food Microbiology, vol. 255, pp. 1-6, (2017).

[16] D. Ercolini, High-throughput sequencing and metagenomics: moving forward in the culture-independent analysis of food microbial ecology, Applied and Environmental Microbiology, vol. 79, pp. 3148-3155, (2013).

[17] S. Ceuppens, D. Li, M. Uyttendaele et al., Molecular methods in food safety microbiology: interpretation and implications of nucleic acid detection, Comprehensive Reviews in Food Science and Food Safety, vol. 13, no. 4, pp. 551-577, (2014).

[18] D. I Ellis, H.Muhamadali, D. P. Allen, C. T Elliott, R.Goodacre, A flavour of omics approaches for the detection of food fraud, Current Opinion in Food Science, vol.10, pp. 7-15, (2016).

[19] A. Galimberti, A. Bruno, V. Mezzasalma, F. De Mattia, I. Bruni, and M. Labra, Emerging DNA-based technologies to characterize food ecosystems, Food Research International, vol. 69, pp. 424-433, (2015).

[20] R. Ferrise, G. Trombi, M. Moriondo, and M. Bindi, Climate change and grapevines: a simulation study for the mediterranean basin, Journal of Wine Economics, pp. 1-17, (2014).

[21] F.R.Pinu, Metabolomics - the new frontier in food safety and quality research, Food Research International, vol. 72, pp. 80-81, (2015).

[22] L.L. Monti, C.A. Bustamante, S. Osorio et al., Metabolic profiling of a range of peach fruit varieties reveals high metabolic diversity and commonalities and differences during ripening, Food Chemistry, vol. 190, pp. 879-888, (2016).

[23] V. Gallo, P. Mastrorilli, I. Cafagna et al., Effects of agronomical practices on chemical composition of table grapes evaluated by NMR spectroscopy, Journal of Food Composition and Analysis, vol. 35, no. 1, pp. 44-52, (2014).

[24] R. Godelmann, F. Fang, E. Humpfer et al., Targeted and nontargeted wine analysis by $1 \mathrm{H}$ NMR spectroscopy combined with multivariate statistical analysis. Differentiation of important parameters: grape variety, geographical origin, year of vintage, Journal of Agricultural and Food Chemistry, vol. 61, no. 23, pp. 5610-5619, (2013). 
[25] V. David, S. Terrat, K. Herzine et al., High-throughput sequencing of amplicons for monitoring yeast biodiversity in must and during alcoholic fermentation, Journal of Industrial Microbiology \& Biotechnology, vol. 41, no. 5, pp. 811-821, (2014).

[26] J. L. Gonçalves, J. A. Figueira, F. P. Rodrigues et al., A powerful methodological approach combining headspace solid phase microextraction, mass spectrometry and multivariate analysis for profiling the volatile metabolomic pattern of beer starting raw materials, Food Chemistry, vol. 160, pp. 266-280, (2014).

[27] A. B. Uryupi, A.S. Peregudov, Application of NMR techniques to the determination of the composition of tobacco, coffee, and tea products, Journal of Analytical Chemistry, vol. 68(12), pp. 1021-1032, (2013).

[28] B. Mayo, C. T. C. C Rachid, Á. Alegría, A. M. O. Leite, R. S. Peixoto, and S. Delgado, Impact of Next Generation Sequencing Techniques in Food Microbiology, Current Genomics, vol.15, pp.293-309, (2014).

[29] E. Dugat-Bony, C. Straub, A. Teissandier, D. Onésime, V. Loux, C. Monnet, F. Irlinger, S. Landaud, M. N. Leclercq-Perlat, P. Bento, et al., Overview of a surface-ripened cheese community functioning by metaomics analyses, PLoS One, 10:e0124360, (2015).

[30] F. De Filippis, A. Genovese, P. Ferranti, J. A. Gilbert, D. Ercolini, Metatranscriptomics reveals temperature-driven functional changes in microbiome impacting cheese maturation rate, Scientific Reports, vol.6, pp. 1-12, February (2016).

[31] E. Sattin, N.A. Andreani, L. Carraro, R. Lucchini, L. Fasolato et al. A Multi-Omics Approach to Evaluate the Quality of Milk Whey Used in Ricotta Cheese Production, Front Microbiol., vol. 17(7), pp. 1272, (2016).

[32] P. J. Fabres, C. Collins, T. R. Cavagnaro and C.M. Rodríguez López, A Concise Review on Multi-Omics Data Integration for Terroir Analysis in Vitis vinifera, Front. Plant Sci., (2017).

[33] M.G. Noel, C. B. Lebrilla, and E. V. Garcia, A Multi-Omics Approach to Finding Biomarkers in Philippine Civet Coffee, in Proceeding De La Salle University, Manila, Philippines, March 7-9, (2016).

[34] A.H. Havelaar, S. Brul, A. de Jong, M. H. Zwittering, B. H. Ter Kuile, Future challenges to microbial food safety, Int J Microbiol, vol.139 (Suppl), pp. 79-94, (2010).

[35] G. Picariello G, G. Mamone, F. Addeo, P. Ferranti, Novel mass spectrometry-based applications of the 'omics' sciences in food technology and biotechnology, Food Technol Biotechnol., vol. 50(3), pp. 286305, (2012).

[36] R. Russo R, V. Severino, A. Mendez, J. Liberia, A. Parente, A. Chambery, Detection of buffalo mozzarella adulteration by an ultra-high performance liquid chromatography tendem mass spectrometry methodology, J Mass Spectrom, vol. 47, pp. 1407-1414, (2012).

[37] T. Briese, et al., Genetic detection and characterization of Lujo virus, a new hemorrhagic fever-associated arenavirus from southern Africa, PLoS Pathogens, vol. 5:e1000455, (2009).

[38] G. J. Baillie, et al., Evolutionary dynamics of local pandemic H1N1/2009 influenza virus lineages revealed by whole-genome analysis, J Virol. , vol. 86, pp. 11-8, (2012).

[39] EFSA (European Food Safety Authority) Report Omics Technologies in Risk Assessment, October (2013).

[40] T. M. Bergholz, A. I. M. Switt, and M. Wiedmann, Omics approaches in food safety: fulfilling the promise? Trends Microbiology, vol.22, pp. 275-281, May (2014).

[41] X. Yang, N. R. Noyes, E. Doster, J. N. Martin, L. M. Linke, R. J. Magnuson, H. Yang, I. Geornaras, D. R. Woerner, K. L. Jones, Use of metagenomic shotgun sequencing technology to detect foodborne pathogens within the microbiome of the beef production chain, Applied and Environmental Microbiology, vol. 82, pp. 2433-2443, (2016).

[42] S. R. Leonard, M. K. Mammel, D. W. Lacher, C. A. Elkins, Application of metagenomic sequencing to food safety: detection of shiga toxin-producing Escherichia coli on fresh bagged spinach, Applied and Environmental Microbiology, vol. 81, no. 23, pp. 8183-8191, (2015).

[43] A. Guarino, A. Giannattasio, New molecular approaches in the diagnosis of acute diarrhea: advantages for clinicians and researchers, Current Opinion in Gastroenterology, vol. 27, pp. 24-9, (2011).

[44] S.P. Liu, J.X. Yu, X.L. Wei, Z.W. Ji, Z.L. Zhou, et al., Sequencing-based screening of functional microorganism to decrease the formation of biogenic amines in Chinese rice wine, Food Control, vol. 64, pp. 98-104, (2016).

[45] A.M. Walsh, F. Crispie, K. Kilcawley, O. O'Sullivan, M.G. O'Sullivan, et al., Microbial succession and flavor production in the fermented dairy beverage kefir, mSystems, (2017).

[46] S. Chakravorty, S. Bhattacharya, A. Chatzinotas, W. Chakraborty, D. Bhattacharya, R. Gachhui, Kombucha tea fermentation: microbial and biochemical dynamics, Int. Jornal Food Microbiooly, vol 220, pp.63-72, January 2016. 
[47] C. Kocharunchitt, et al., Integrated transcriptomic and proteomic analysis of the physiological response of Escherichia coli O157:H7 Sakai to steady-state conditions of cold and water activity stress, Mol Cell Proteomics, vol. 11, (2012).

[48] V. García-Cañas, C. Simó, M. Herrero, E. Ibáñez, A. Cifuentes, Present and future challenges in food analysis, Foodomics, Anal Chem, vol 84, pp. 10150-10159, (2012).

[49] C. Ibáñez, A. Valdés, V. García-Cañas et al., Global Foodomics strategy to investigate the health benefits of dietary constituents, Journal of Chromatography A, vol. 1248, pp. 139-153, (2012).

[50] Y. Hasin, M. Seldin, A. Lusis, Multi-omics approaches to disease, Genome Bioliology, vol. 18, pp. 83, (2017).

Citation: T. M. Sirangelo, "High-Throughput Sequencing Technologies and new Approaches in Food Microbiology ", International Journal of Research studies in Microbiology and Biotechnology, vol. 4, no. 1, p. 44-51, 2018. http://dx.doi.org/10.20431/2454-9428.0401006

Copyright: (c) 2018 Authors. This is an open-access article distributed under the terms of the Creative Commons Attribution License, which permits unrestricted use, distribution, and reproduction in any medium, provided the original author and source are credited. 